\title{
Safety Monitoring of Expressway Construction Based on Multisource Data Fusion
}

\author{
Dianliang Xiao, ${ }^{1}$ Tiantao Zhang, ${ }^{2}$ Xudong Zhou, ${ }^{3}$ Guangshun Zheng, ${ }^{2}$ and Haoran Song ${ }^{1}{ }^{1}$ \\ ${ }^{1}$ Transportation Safety Research Center, China Academy of Transportation Sciences, Beijing 100029, China \\ ${ }^{2}$ Qilu Transportation Development Group, Jinan 250014, Shandong, China \\ ${ }^{3}$ Guangdong Highway Construction Co., Ltd., Guangzhou 510699, Guangdong, China \\ Correspondence should be addressed to Haoran Song; songhr@motcats.ac.cn
}

Received 16 June 2020; Revised 14 July 2020; Accepted 31 July 2020; Published 1 September 2020

Academic Editor: Sang-Bing Tsai

Copyright (c) 2020 Dianliang Xiao et al. This is an open access article distributed under the Creative Commons Attribution License, which permits unrestricted use, distribution, and reproduction in any medium, provided the original work is properly cited.

\begin{abstract}
China's terrain is complex, both plain, microhill (heavy-hill) and mountainous terrain; the hidden dangers of highway construction are prominent. Construction site management, production safety management, and construction personnel management are difficult, and it is necessary to borrow advanced technology to establish information, and it is necessary to borrow advanced technology to establish information system to realize the visualization of safety monitoring. In the construction of highways, mountainous terrain is often complicated due to complex terrain, high mountains, and deep valleys. Excavation of the mountain mass is required to form high and steep slopes. For successful projects, safety monitoring is particularly important. Multisource data fusion is one of the computer application technologies. It is an information processing technology that is automatically analyzed and synthesized under certain criteria to complete the required decision-making and evaluation tasks. This paper analyzes high-speed data in the context of multisource data fusion. Study on highway slope construction safety monitoring. BP neural network fusion technology of multisource data fusion technology is used. A high-speed breccia-bearing silty clay slope is taken as the research object. The feedback information about the deployed monitoring system is fully used in the slope design and construction. The construction design parameters are reversed to predict the stability of the slope and ensure the safety of construction and operation of similar slopes of the entire expressway. The research in this paper finds that the maximum deviation between the slope displacement value and the measured value obtained by the slope monitoring based on multisource data fusion in this paper is $7.53 \%$, which is less than $10 \%$, which verifies the feasibility of the method in this paper. The research methods and ideas of this paper can also provide a reference for similar engineering research.
\end{abstract}

\section{Introduction}

At present, general highway construction project companies and higher-level units cannot implement real-time, comprehensive, and image-based safety monitoring of construction sites due to traffic and environmental reasons: construction management efficiency is not high and managers are struggling with high-load and high-intensity inspections on construction sites or unannounced inspections; many safety management actions are ex post facto. Generally, it is not until a hidden safety hazard appears or a safety accident occurs after some time that it is discovered. It may even take some time for the safety problem to be effectively corrected. The existing methods are difficult to implement real-time command on the construction site, which requires the personnel of all parties to be concentrating on the site. It lacks intuitive on-site video data, and it is difficult to supervise the work of the supervision station, which will inevitably affect the analysis of the cause of the accident and the division of responsibilities. Safety production management is difficult and has also gradually increased. Under some occasions, certain environments, and other major dangerous sources, there is a dire need to use advanced science and technology to establish real-time traceable dynamic engineering archives and information-oriented construction site safety monitoring systems, to provide powerful technical means to improve the work efficiency of on-site management personnel, reduce the probability of 
accidents in highway construction, and create a "safe construction site" through visualization and information technology, which can provide a strong guarantee for the safe production and emergency rescue of engineering projects.

The highway construction project has the characteristics of many construction points, long lines, remote geographical locations, inconvenient transportation, and many units participating in the construction. The hidden safety hazards are particularly prominent [1]. Nowadays, the proportion of bridges and tunnels on expressways is generally high, and there are many key projects such as long tunnels, high pier bridges, and high slopes, such as a large number of waterworks, cross-road bridges, cross-rail bridges, and overpasses; the terrain and geological conditions are complex and result in extensive Karst development, rich groundwater, faults, and other bad geology, complex construction site management, difficult construction conditions, and difficult access roads [2]. Slopes are used to protect the stability of the roadbed. Slopes with a certain slope are made on both sides of the roadbed. However, under certain conditions, landslides and collapses may occur. Buried houses increase huge operation and maintenance costs, so the monitoring of slopes in highway construction safety monitoring is of great significance to ensure the safe operation of highways $[3,4]$.

HUANG Yong used the support mechanism of the high slope of the roadway in the highway reconstruction project and optimized the support scheme. Taking the left side of the K1415 + 200 high slope on the Liunan (Liuzhou to Nanning) highway as an example, the FLAC3D numerical method was first used. The influence of slope excavation on the stability of the slope and then an orthogonal test was designed to analyze the influence of anchor angle, anchor length, and anchor spacing on the safety factor of the slope during anchor support. In another study, Huang Yong changed slope safety factor under the conditions of antislide piles and excavation before the original slope construction. Monitoring points were set by the slope height. The author analyzed the horizontal and vertical displacement, and the stress of slope soil was studied with a strain increment cloud map. The results indicate that although the author's method is difficult to construct, it is economical and safe and its coefficient is high. Finally, the support effects of the two support schemes were compared in his study $[5,6]$. The representative design elements of road geometry are longitudinal slope, flat curve radius, superelevation, and gentle curve. The design criteria of these elements are combined in various ways according to the function of the road. WU Hai-bao divided highways into homogeneous sections based on longitudinal slopes and flat curve radii. The author matched the data required for analysis of various links and used the established data to establish a safety performance function. Finally, a collision correction factor that could explain the exposure rate of traffic accidents was calculated. When the threshold of the horizontal curve radius $R=1000 \mathrm{~m}$ is set to 1.0 , the collision correction coefficient of $R=300 \mathrm{~m}$ is calculated as
1.33 , and the accident exposure rate is increased by $33 \%$. When the critical $0 \%$ threshold is set to 1.0 , the collision correction coefficient indicates that the accident exposure rate decreases on the uphill and rises on the downhill. The research results can be used as the basic data for the geometric design of expressways during the improvement period $[7,8]$. Yang et al. targeted the current situation of single safety monitoring information for construction projects, slow information feedback analysis, and the inability of participants to work together. The proposed system was also suitable for modern information technology and monitoring indicators and control points of the existing construction projects, to obtain comprehensive information on monitoring targets, including building entity information, construction site environmental information, and security ontology knowledge information. From the perspective of information collaboration, Yang et al. proposed a security monitoring information collaboration system for construction projects based on the integrated information of the data warehouse integrated monitoring target, including an information acquisition module, information processing module, and information collaboration module. All the participants can work together based on the security information control center and finally realize real-time visualization, automation, and informationization of security monitoring $[9,10]$. Okumura et al. introduced the research status of ACSDINS based on analyzing the background and current research status of building safety monitoring. The system used acoustic vector sensors as the main equipment to realize signal acquisition and selected steel mold as the monitoring object. Okumura et al.'s experiments and analysis results proved the feasibility of ACSDINS. This research result was broadly applied and had important practical significance in the field of safety monitoring [11, 12].

The present study uses the BP neural network data fusion method in multisource data fusion technology to monitor and study slope safety in highway construction safety. In this paper, the data of field monitoring points are substituted into the inversion system for parameter inversion. The slope model at the monitoring points is established based on the inversion results. The analysis and calculation are compared with the measured data of the monitoring points. It is $7.53 \%$ and less than $10 \%$. It indicates that the mechanical parameters of breccia-bearing silty clay obtained through inversion are reasonable and can be used as predictive analysis parameters of the slope stability.

\section{The Proposed Method}

\subsection{Multisource Data Fusion Technology}

2.1.1. Multisource Data Fusion. Multisource data fusion is also called multisensor information fusion [13]. Multisource data fusion technology studies how to integrate multisource data information or related auxiliary data to obtain more accurate and reliable results than using a single data [14]. It is 
an information processing technology that is automatically analyzed and integrated under certain criteria to complete the required decision-making and evaluation tasks. The cognitive process of objective things by humans and other animals in nature is a process of fusion of multisource information.

Multisource data fusion is divided into data-level fusion, feature-level fusion, and decision-level fusion according to the level of fusion [15]. Data-level fusion is the lowest level of fusion. It directly performs fusion processing on the sensor's observation data and then performs feature extraction and judgment decisions based on the fusion result. For example, in imaging sensors, the sensor performs image processing on a blurred image containing a certain pixel. The process is to confirm target attributes. This level of fusion is mostly used for image analysis and direct synthesis of similar radar waveforms [16].

Feature-level fusion refers to the extraction of a set of feature information from the original information provided by each sensor to form a feature vector, and the fusion of each group of information before classifying or other processing of the target, sometimes called intermediate-level fusion, a commonly used method. There are cluster analysis methods, artificial neural networks, and K-order nearest neighbor methods.

(1) Data Layer Fusion. Data layer fusion is a level of fusion that uses the direct data collected by the sensors to perform preliminary analysis and integration based on expert experience and description [17]. The main advantage of this fusion is that it processes first-level data, including proofreading and identification, analysis, and synthesis, and the resulting results can be imported into the database, can be used by system users, and can provide accuracy that cannot be provided by other fusion levels. However, its shortcomings are also obvious. Because the raw data of all sensors is collected, the system needs more storage space, the central processor that needs to process the raw data is more demanding, and the system software needs more powerful error correction capabilities. Both require high costs. This fusion level is mainly used for the synthesis, analysis, and organization of the initial image data collected by various inspection methods. Data layer fusion is shown in Figure 1.

From Figure 1, the data layer fusion first collects data information from multiple sensors, filters out the associated data information and passes it to the data layer for fusion, performs feature extraction and attribute judgment, and finally performs joint attribute judgment to output relevant data.

(2) Feature Layer Fusion. Feature-level fusion uses the feature information collected by sensors at various levels to improve target recognition capabilities. Using feature-level fusion algorithms, efforts are made to combine different types of fault features to make a comprehensive judgment [18]. The characteristic factors used in this system are as follows: connection layer, normalization, number of components, spatial information, and multiscale. Compared with a single feature recognition system, this fusion system has higher accuracy and stability and will be more widely used in applications. Feature layer fusion mostly adopts a distributed or centralized fusion structure. The specific model is shown in Figure 2.

Figure 2 demonstrates that feature layer fusion refers to extracting locally representative data information from different sensors, then combining these local data to obtain vectors with significant features.

(3) Decision-Level Integration. Decision-level fusion requires a database and expert decision-making system to analyze and reason about raw data or intermediate-level data to obtain expert-level decision-making results. This method requires a large amount of calculation, and the use of expert decision-making systems will bring high costs [19]. Decision-level fusion is a high-level fusion. The specific model is shown in Figure 3.

Figure 3 illustrates that the preliminary conclusions on the object are formed after the basic processing operations, such as preprocessing, feature extraction, and judgment and recognition, are performed on the information of different types of sensors locally. The fusion center then performs further fusion processing on the local decision results. Because the decision-making layer is the overall decision result obtained by fusion processing based on the decision results of each sensor.

2.1.2. Information Fusion Method. With the development of sensor technology, information fusion technology is also developing rapidly. Many scholars proposed a variety of effective information fusion methods, which are summarized as weighted average method, D-S evidence reasoning algorithm, Bayesian reasoning algorithm, Mann filter method, fuzzy logic reasoning algorithm, artificial neural network algorithm [20]. Each method has different advantages and disadvantages and its application scope. In practical applications, corresponding algorithms should be selected for different situations.

2.1.3. Process of Information Fusion. The process of information fusion mainly includes the following five categories:

(1) Data Input/Data Output (DAI-DAO). This is the most basic and lowest-level form of fusion, which belongs to datalevel fusion. This process is mainly the fusion processing of the raw data input from multiple information sources, such as traffic flow data preprocessing.

(2) Data Input/Feature Output (DAI-FEO). This process belongs to data-level fusion, and its main function is to extract the traffic flow state characteristic information of the target road section through the fusion of multisource data according to the needs of solving practical problems.

(3) Feature Input/Feature Output (FEI-FEO). This process belongs to feature-level fusion, and its main function is to analyze and merge the spatial and temporal characteristics of the extracted traffic flow state characteristics of the target road section. 


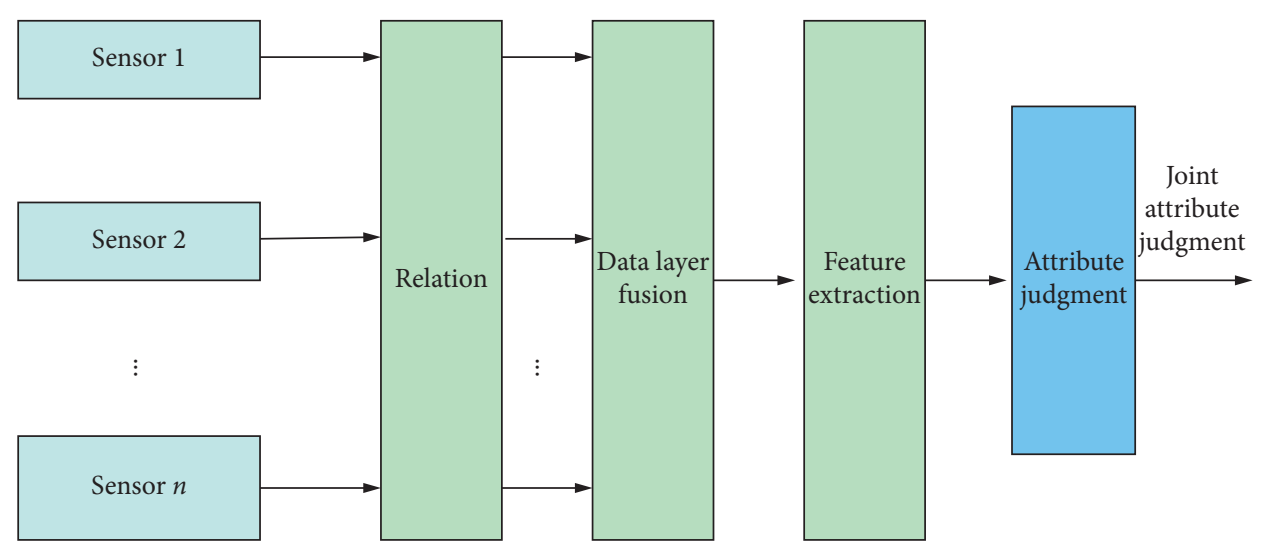

Figure 1: Data layer fusion structure.

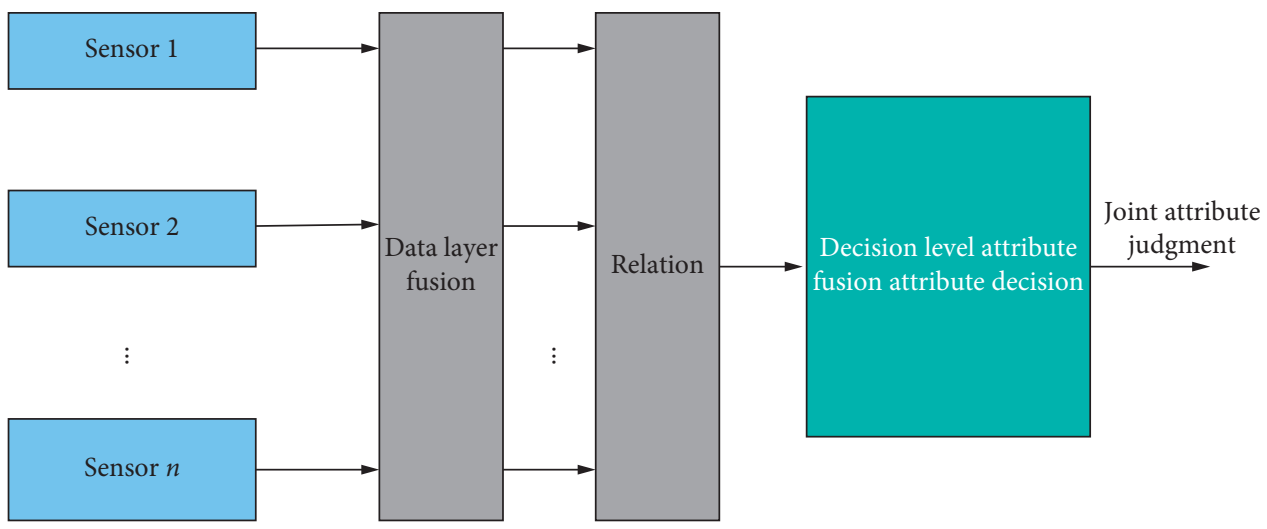

Figure 2: Feature layer fusion structure.

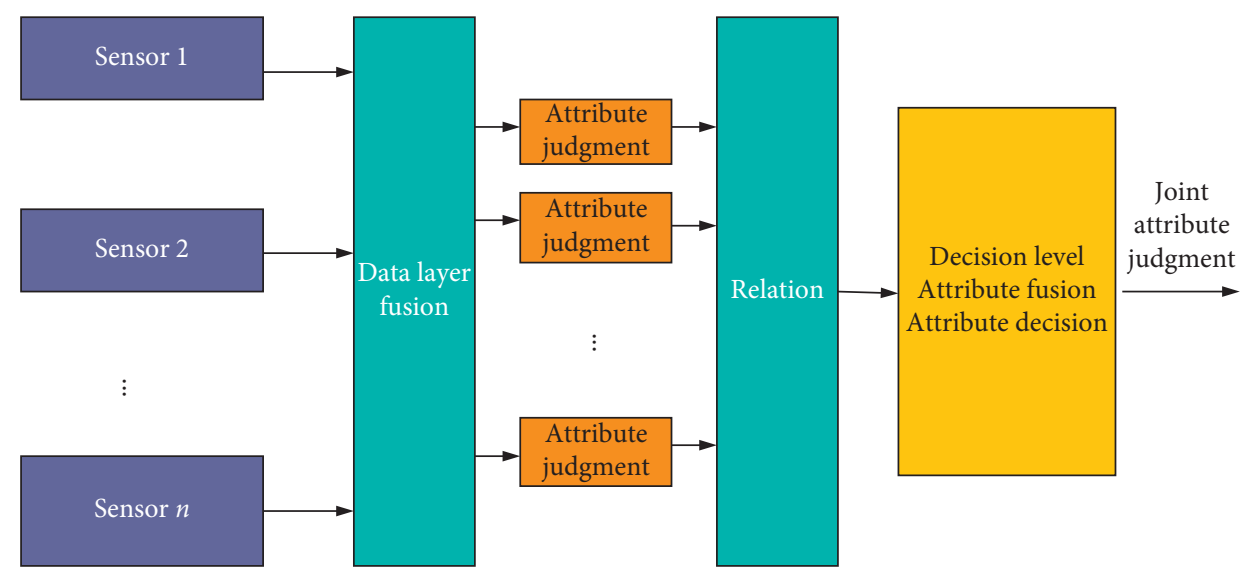

Figure 3: Decision-level fusion structure.

(4) Feature Input/Decision Output (FEI-DE). This process is a decision-level fusion. Based on the characteristics of the traffic flow state of the existing target road segment, the classification result is obtained by classifying and identifying the characteristics from different information sources.

(5) Decision Input/Decision Output (DEI-DEO). This process belongs to decision-level fusion. Its main function is to further fuse local decision-making results, making the final fusion result more accurate and reliable.

\subsection{Multisource Data Fusion Model}

2.2.1. First-Level Data Fusion Method. At present, the most commonly used method for preprocessing the raw data is the weighted average method [21]. The weighted average fusion algorithm uses a mathematical average formula, which is difficult to overcome the influence of some sensor failures on the measured values and the measurement speed memory. Based on the weighted average algorithm, the optimal weighting fully considers the influence of each sensor system 
on the measurement data and has a good strain handling ability for some sensor failures in the system; and the optimal weighted fusion algorithm is used to fuse the measurement data only. There is a need to know the variance of the sensor's measurement noise.

\subsubsection{The Estimation Model of Optimal Weighted Fusion.} For the measurement equation of the sensor, the mean square error is often used as the evaluation criterion for the fusion result. The optimal weighted fusion is to find the minimum mean square error. Multiply the measurement data of each sensor by a certain weight coefficient and add them. The results of data fusion are in [22]. When $n$ sensors detect the same index parameter at different positions, the measurement equation of the $i$ sensor is

$$
Y_{i}=X+M_{i}, \quad i=1,2, \ldots, n .
$$

Among them, $X$ is the true value of the estimated parameter, $Y_{i}$ is the measurement value, and $M_{i}$ is the measurement noise. The measurement noise of each sensor is independent of each other. Let each sensor's weighting factor be $W_{i}(i=1,2, \ldots, n)$ and then get

$$
\begin{aligned}
\widehat{X} & =\sum_{i=1}^{n} w_{i} \cdot Y_{i}, \\
\sum_{i=1}^{n} w_{i} & =1 .
\end{aligned}
$$

The mean square error of the weighted estimate is

$$
\sigma^{2}=E[(X-\widehat{X})]=\frac{1}{\sum_{i=1}^{n} \sigma_{i}^{2}} .
$$

It can be known from the above formula that the smaller the measurement noise variance is, the larger the sensor weight is, and the proportion of the corresponding measurement data in the weighted estimation value is higher; on the contrary, the larger the sensor noise value is, the smaller the weight is, and the measurement data is in the weighted estimation. The proportion in the value is low. The mean square error of the optimal weighted estimate is less than the variance of the measurement noise of any sensor in the system.

2.2.3. BP Neural Network Two-Level Fusion Model. An artificial neural network, which is also referred to as a neural network, is a research area used to simulate the structure and intelligence of the human brain. An important feature of it is that the output of the network is consistent with expectations through network learning ability $[23,24]$. BP neural network is a feedforward network and is one of the most important models in ANN. It can be used to approximate any nonlinear continuous function with arbitrary accuracy and has good self-adaptation and fault tolerance in nonlinear systems.

(1) BP Neural Network Structure. Mostly, BP neural network has one input layer and one output layer, one or several hidden layers. Each layer is connected, and neurons in the same layer are not connected. The multilevel network architecture can ensure that the BP network can obtain more comprehensive information from the input layer and then can handle more complicated information. The structure of the BP neural network is shown in Figure 4.

$X_{1}, X_{2}, \ldots, X_{n}$ are input values of the network, $Y_{1}, \ldots, Y_{n}$ are output values of the network, and $W_{i j}$ and $W_{j k}$ are weights of the neural network. When the number of input nodes is $n$ and the number of output nodes is $m$, a function mapping relationship from $n$ independent variables to $m$ dependent variables is formed.

(2) BP neural network training is required to give the network storage memory and prediction ability before BP network prediction. $B P$ network training process:.

Step 1. The first step defines network initialization. According to the network input and output sequence $(X, Y)$, select the number of input layer nodes $n$; the number of hidden layer nodes is 1 , and the number of output layer nodes is $m$. Initially, the connection weight between the input layer and the hidden layer is $W_{i j}$, and the connection weight between the hidden layer and the output layer is $W_{j k}$, the hidden layer threshold is initialized to $a$, and the initial output layer threshold is set to $b$. At the same time, the learning rate and neuron excitation function is assigned.

Step 2. Hidden layer output calculation: according to the input variable $X$, the connection weight is $W_{i j}$ between the input layer and the hidden layer, and for the hidden layer threshold $a$, the output $H$ of the hidden layer is calculated:

$$
H_{j}=f\left(\sum_{i=1}^{n} w_{i j} x_{i}-a_{j}\right), \quad j=1,2, \ldots, I .
$$

In the formula, $I$ is the number of nodes in the hidden layer and $f$ is the excitation function of the hidden layer.

$$
f(x)=\frac{1}{1+e^{-x}} .
$$

Step 3. Output layer output calculation: according to the output $H$ of the hidden layer, the connection weight is $W i j$ between the hidden layer and the output layer, and for the output layer threshold $b$, the predicted output $O$ of the BP neural network is calculated:

$$
O_{k}=\sum_{j=1}^{I} H_{j} w_{j k}-b_{k}, \quad k=1,2, \ldots, m .
$$

Step 4. Error calculation: according to the predicted output $O$ and expected output $Y$ of the network, the prediction error $e$ of the neural network is calculated:

$$
e_{k}=Y_{k}-O_{k}, \quad k=1,2, \ldots, m
$$




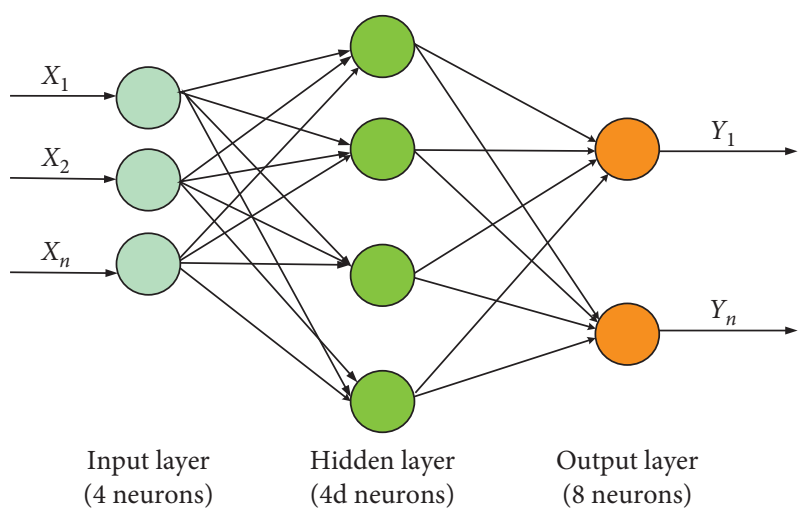

FIGURE 4: Structure of BP neural network.

Step 5. Weight update: update the network connection weights wij, wjk according to the prediction error $e$ of the neural network:

$$
w_{j k}=w_{j k}+\eta H_{j} e_{k}, \quad j=1,2, \ldots, I ; k=1,2, \ldots, m,
$$

where $\eta$ is the learning rate.

Step 6. Threshold update: update the network node thresholds $a$ and $b$ according to the prediction error $e$ of the neural network:

$$
a_{j}=a_{j}+\eta H_{j}\left(1-H_{j}\right) \sum_{k=1}^{m} w_{j k} e_{k}, \quad j=1,2, \ldots, I .
$$

Step 7. According to the requirements of the condition, determine whether the algorithm iteration ends or not. If not, return to the second step.

(3) Multilayer Network Design Based on BP Algorithm. Multilayer network design based on the BP algorithm has different layers of the hidden layers, which can be divided into single hidden layers and multiple hidden layers [107108]. Compared with a single hidden layer, a multihidden layer has stronger generalization ability and higher prediction accuracy, but the disadvantage is that it takes longer training time. Therefore, the choice of the number of hidden layers should be considered from the accuracy and time. For complex mapping relationships, multiple hidden layers can be selected to improve the prediction accuracy. For the recognition of general patterns, the accuracy can meet the requirements, the choice of a single hidden layer. A threelayer network can be well solved, speed up, and save time. The information capacity of the neural network (the weight of the neural network and the total number of thresholds) has a significant impact on the actual application of the network and is closely related to the number of training samples and training errors, as shown in the following formula:

$$
P \approx \frac{n_{w}}{\varepsilon}
$$

In the formula, $P$ is the number of training samples and is the training error and $w n$ is the total number of weights and thresholds of the neural network. Therefore, under the condition of guaranteed training error, when the training samples are not enough, the information capacity of the neural network should be appropriately reduced by reducing the sample dimension.

\subsection{In Situ Monitoring of Site Displacement during Highway Slope Construction}

2.3.1. Slope Displacement Monitoring and Burying Design of Inclined Pipe. Expressway slopes are permanent slopes that are in service during operation. To ensure the safety of the slope, prevent slope instability and damage, and ensure the smooth passage of high-speed sections, the slope of the highway must be monitored for a long period. Obtain the stability of the slope for timely construction control and remediation [25].

(1) Monitoring purpose: the slope monitoring can be mainly used for the following two points: First, data monitoring during the construction period will guide the data results to the construction and feedback of the construction design. Secondly, based on the monitoring data of the longer observation period of the construction period, relevant geotechnical parameters, protection measures design plan, and other data, the slope stability is analyzed and calculated, and relevant reasonable suggestions are provided for maintenance and repair work during the operation period.

(2) The monitoring points are based on the actual geological conditions of the slopes of the Chang'an Expressway and the characteristics of the existing structures and carry out horizontal displacement monitoring of the slope surface, deep displacement monitoring of the slope body, vertical displacement (subsidence) monitoring of the slope surface, groundwater level monitoring, and so forth four items. This monitoring plan is designed to arrange a total of 12 observation points for horizontal displacement of the slope, 6 monitoring points for vertical displacement (settling) on the slope surface, and 6 monitoring points for the groundwater level.

2.3.2. Principle of Inclined Pipe and Slope Excavation Displacement and Water Level Monitoring. Inclinometer can measure the internal lateral displacement of rock and soil with high accuracy and is widely used for in situ monitoring of slope engineering [26]. The inclinometer consists of a probe, a cable, and a reader. Before monitoring, the inclinometer pipe must be buried in the rock and soil layer in advance, and the guide roller card of the instrument can be moved up and down in a symmetrically distributed groove in the pipe. When measuring, the sensing direction of the instrument needs to be aligned with the sliding direction of the slope, so that the sensor slowly slides into the bottom of 
the tube, and then remains stable for 5 to 10 minutes, so that the temperature of the sensor is close to the external underground temperature, then lifting the cable from the bottom of the measuring tube. The distance to the measuring nozzle is measured at a distance of $0.5 \mathrm{~m}$ and is recorded after the reading of the reading instrument is stable until the nozzle position, and then the reverse measurement is performed in the same way, and the corresponding data value is read and recorded. The inclinometer works. The principle is to use the internal pendulum of the sensor probe affected by gravity to measure the internal angle between the sensor and the plumb line and then calculate the gap between the hole axis and the plumb line by geometric theory. The horizontal displacement is calculated, and then, it is accumulated to obtain the total displacement and the deep displacement change in the entire hole along the axis of the tube.

When measured with an inclinometer, the error caused by the instrument itself and external influences should be reduced as much as possible. The forward displacement and reverse measurement should be used to measure the deep soil displacement of the slope once, and the measured value should be taken twice, mean of algebraic differences.

The MCU remote monitoring system can realize the automatic monitoring of surface displacement. Both the surface displacement monitoring data and the slope water level monitoring data can be transmitted to the computer through the instrument, and then the data can be summarized and processed for slope stability analysis and research.

2.3.3. Processing Method of Slope Monitoring Data. Obtain soil deformation monitoring data through a borehole inclinometer and then arrange the deformation process curve. Demonstrate the changes and current status of deformation traits. The monitoring results are comprehensively analyzed from space, time, and environmental factors to find the cause of curve deformation, accurately explain the deformation curve, identify the stability of the soil, provide a basis for design, construction, and engineering treatment, and provide reference data for forecasting.

\subsubsection{Causes of Error in Monitoring Data and Avoiding} Measures. In slope monitoring, the occurrence of errors will inevitably affect the reliability of the inclinometer monitoring results, analyze the errors of each link, and establish a scientific and reasonable error elimination method, which will directly affect the scientificity and feasibility of the monitoring results [27]. Any part of the application of the inclinometer instrument may produce errors. The inclinometer itself may have errors: offset error, zero-point drift error, and rotation error; the inclinometer tube may cause distortion errors of the tube during manufacture and installation; during the observation process, it may occur due to different ambient temperature and humidity, environmental errors; human errors are like errors in reading values, misreading values, and misremembering values; calculation errors may also occur during data processing. The above possible errors will affect the accuracy of the subsequent
TABLE 1: Important functions and functions of some BP Networks.

\begin{tabular}{lc}
\hline Function name & Corresponding function \\
\hline tansig & S-type transfer function \\
purelin & Linear transfer function \\
logsig & Logarithmic S-type transfer function \\
deltatan & Delta function of tansig neurons \\
deltalog & Delta function of logsig neurons \\
learnbp & BP learning rules \\
\hline
\end{tabular}

displacement inversion analysis and soil parameter inversion analysis results. Therefore, during the monitoring process, the error caused by the monitored monitoring data must be accurately and reasonably monitored to minimize the monitoring error.

\section{Experiments}

3.1. Training Sample Preprocessing. Before the BP neural network performs prediction, the input and output data need to be normalized. The data is transformed to $[0,1]$ or $[-1,1]$. The purpose is to eliminate the order of magnitude difference between the data in each dimension, so that each component of the neural network has the same important status, which can effectively prevent the network prediction error from being too large due to the large order difference. The formula for transforming $[0,1]$ interval of data is as follows:

$$
\bar{X}_{i}=\frac{X_{i}-X_{\min }}{X_{\max }-X_{\min }}
$$

In the formula, $X_{i}$ represents the input or output data, $X_{\min }$ represents the minimum value in the data sequence, and $X_{\max }$ represents the maximum value in the data sequence. The formula for transforming the data $[-1,1]$ interval is as follows:

$$
\bar{X}_{i}=\frac{2 X_{i}\left(X_{\max }+X_{\min }\right)}{X_{\max }-X_{\min }} .
$$

BP network training once runs the training sample forward for one round and reverses the network weight. During the training process, each round of data is best selected in different orders and used repeatedly, usually training up to 10,000 times. The sample set of the network can generally be divided into two parts: one part is used as the training set, which is used for network training; the other part is used as the test set, which is used to test the neural network. To determine whether the neural network has a good generalization ability, it is tested by using a test set. If the generalization ability of the neural network is poor, it expresses that the error is small for the training set but large for the test set. When the number of nodes in the hidden layer of the neural network is constant, there exists an optimal number of times to train, at which time the network generalization ability is optimal. When the number of training times does not reach $t_{0}$, it means that network training is insufficient. After $t_{0}$, network training is excessive. Some important functions and functions of the BP network are shown in Table 1. 
TABLE 2: Parameters of silty clay with breccia.

\begin{tabular}{|c|c|c|c|c|c|}
\hline Rock and soil layer & $\begin{array}{c}\text { Bulk density } \\
\left(\mathrm{KN} / \mathrm{m}^{3}\right)\end{array}$ & Modulus of elasticity (MPa) & Cohesive force $(\mathrm{KPa})$ & Internal friction angle $\left(^{\circ}\right)$ & Poisson's ratio \\
\hline Silty clay with breccia & 19 & $15-27$ & $21.5-30$ & $20-22$ & 0.3 \\
\hline
\end{tabular}

TABLE 3: Inversion value of soil parameters.

\begin{tabular}{lccc}
\hline Inversion parameters & Internal friction angle $\left(^{\circ}\right)$ & Cohesion $(\mathrm{KPa})$ & Modulus of elasticity $(\mathrm{MPa})$ \\
\hline Silty clay with breccia & 21.88 & 29.55 & 26.90 \\
Strongly weathered marl & 34.96 & 49.70 & 117.15 \\
\hline
\end{tabular}

3.2. Selection of Input Layer and Output Layer for Building a Network Model. The measured deep displacement of the slope is monitored at intervals of $0.5 \mathrm{~m}$. Model analysis is performed to calculate the displacement value of the number. 3 oblique tube as a network input sample set. During the trial calculation, it was found that the amount of input caused the network structure. It is too large, the network analysis time is long, and the convergence effect of the network is not obvious. This article improves the network design process and simplifies the network design. Considering that the inputs of each soil layer do not affect each other, the input layer is divided into 9 units, which is denoted as Ii $(i=1,2,3,4,5(6,7,8,9)$ correspond to the displacement values at the monitoring points in the model at $16.5 \mathrm{~m}, 14.5 \mathrm{~m}, 12.5 \mathrm{~m}, 10.5 \mathrm{~m}, 8.5 \mathrm{~m}, 6.5 \mathrm{~m}, 4.5 \mathrm{~m}, 2.5 \mathrm{~m}$, and $0.5 \mathrm{~m}$, the output layer is soil parameters, and the internal friction angle, cohesion, and elastic modulus are separately trained using three networks; the method and principle are the same. Each network has 9 input nodes and 2 output nodes, which greatly simplifies the complexity of the network structure and facilitates network convergence and analysis.

3.3. Experimental Design. The multisource data fusion BP neural network model is used to monitor the safety of the highway slope construction, and the monitoring data is analyzed and arranged. The test object is the construction section of a certain highway slope. The date of monitoring data is selected from mid-October to December.

In the early days, the data of 10 monitoring points were selected for analysis. Table 2 shows the parameters of the breccia silty clay soil on the highway slope construction section.

\section{Discussion}

4.1. Analysis and Comparison of Slope Displacement of Highway Slope Construction Safety Monitoring Based on Multisource Data Fusion. The measured displacement values corresponding to the different depths of the third monitoring point in the field are substituted into the trained BP neural network for inversion to obtain the soil parameters. The parameter inversion values are shown in Table 3. Comparison and analysis of slope displacement calculation analysis and actual measurement of highway safety monitoring based on multisource data fusion are shown in Figure 5 and Table 4.

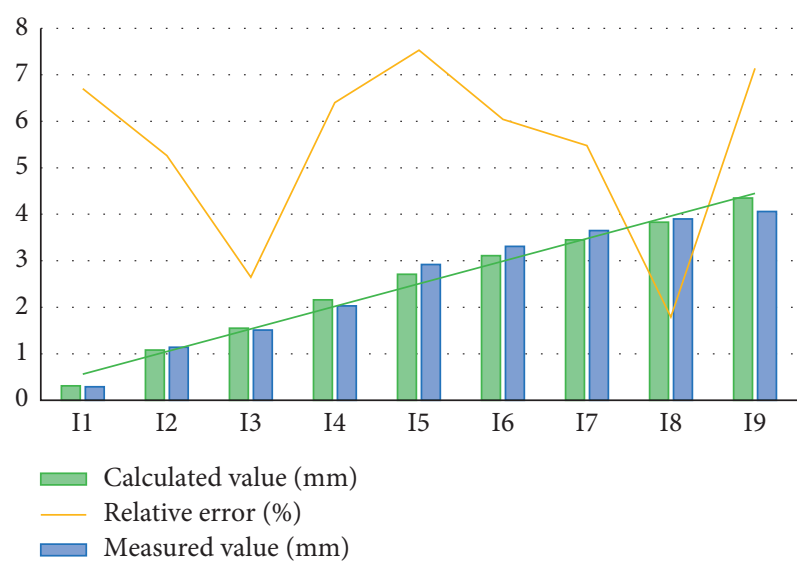

FIGURE 5: Analysis of the comparison between the calculated value and the measured value of the displacement at different depths at the monitoring point.

To make the comparison result more intuitive, the slope construction in which the soil parameters were simulated was inverted, and the comparison between the calculated displacement values at different depths and the measured displacement values at the fourth monitoring point is shown in Figure 6.

Combining Table 4 with Figures 5 and 6, it can be found that the maximum error between the displacement value and the measured value at different depths is $7.53 \%$, which is less than $10 \%$. It is reasonable, and the numerical results can be used for subsequent analysis and research. In addition, by analyzing three different parameters separately, this research method has fewer errors and can be popularized.

4.2. Analysis of Surface Horizontal Displacement Data. The surface displacement value changes continuously with the slope excavation. The surface displacement value is measured before the slope excavation as the initial displacement value. As the excavation progresses, the measured value of the slope displacement is compared with the initial displacement value to obtain the change in displacement. Then, select a part of the monitoring data of 10 monitoring points in the surface displacement monitoring data of a highway from mid-October to early December and make a displacement change trend chart. The results are shown in Figures 7 and 8. 
TABLE 4: Comparison between calculated and measured displacement values at different depths of monitoring points.

\begin{tabular}{|c|c|c|c|c|c|c|c|c|c|}
\hline Measuring point & $\mathrm{I}_{1}$ & $\mathrm{I}_{2}$ & $\mathrm{I}_{3}$ & $\mathrm{I}_{4}$ & $\mathrm{I}_{5}$ & $\mathrm{I}_{6}$ & $\mathrm{I}_{7}$ & $\mathrm{I}_{8}$ & $\mathrm{I}_{9}$ \\
\hline Calculated value $(\mathrm{mm})$ & 0.31 & 1.08 & 1.55 & 2.16 & 2.71 & 3.11 & 3.45 & 3.83 & 4.35 \\
\hline Measured value (mm) & 0.29 & 1.14 & 1.51 & 2.03 & 2.92 & 3.31 & 3.65 & 3.90 & 4.06 \\
\hline Relative error (\%) & 6.70 & 5.26 & 2.65 & 6.40 & 7.53 & 6.04 & 5.48 & 1.79 & 7.14 \\
\hline
\end{tabular}

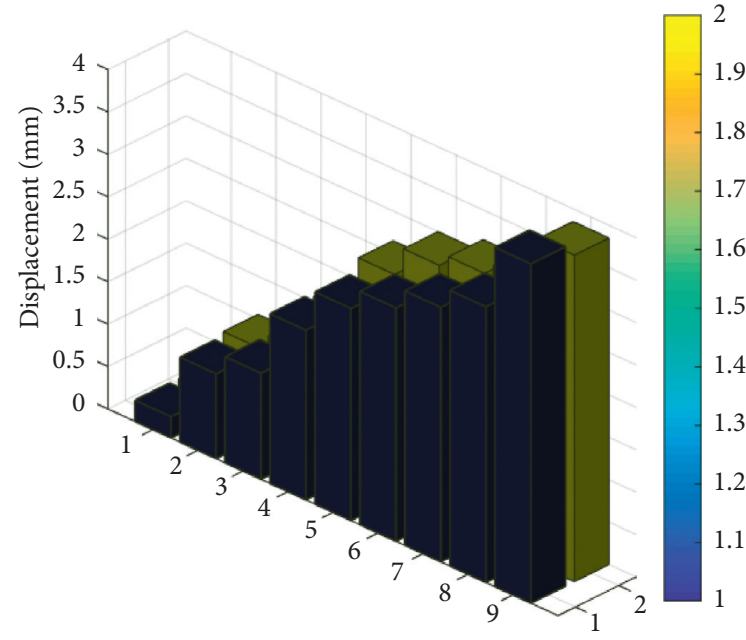

Calculated value Measured value

FIGURE 6: Analysis chart of comparison result between the calculated value and the measured value.

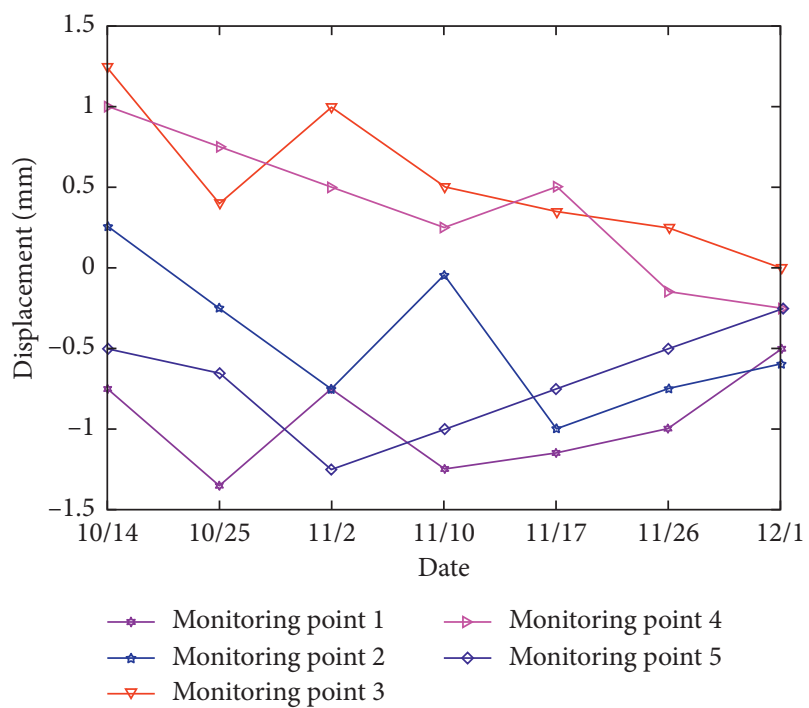

FIGURE 7: Surface displacement trend of monitoring points 1-5.

The value of the analysis of the surface displacement data of the monitored slope is the maximum value of the current displacement on the day and the analysis of the horizontal surface displacement data. The rainy weather and the infiltration of rainwater cause a significant change in the horizontal displacement. When the weather is fine, the soil surface water evaporates and the internal contraction will cause changes in horizontal displacement shrinkage. Months of August and September were in the rainy season, but due to

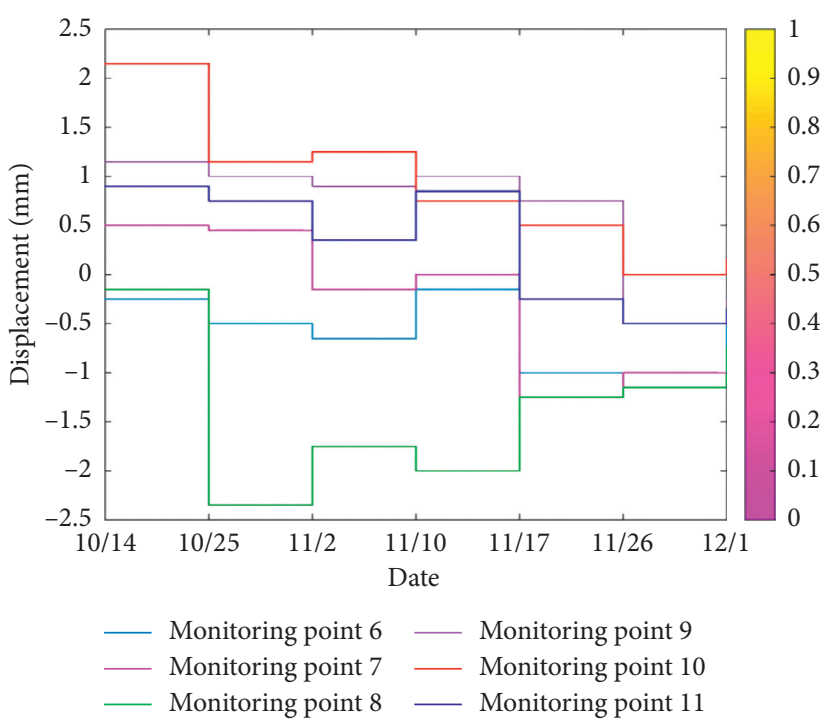

FIGURE 8: Surface displacement trend of monitoring points 6-11.

better drainage facilities and timely drainage, the slope surface displacement did not change significantly. From November to December, weather was mainly sunny. It can be seen from Figures 7 and 8 that with the support of the lower slope of the slope formed, from mid-October to mid-November, the surface displacement of the slope changed a little, without significant fluctuations. By December, the slope displacement stabilized. It is consistent with the actual slope situation.

\section{Conclusions}

With the development of large-scale highway engineering construction, under the requirements of safety production and quality supervision, the construction of a construction monitoring system can provide technical support for the project site and safety management informatization construction. It provides a strong guarantee for the safety production and emergency rescue of the engineering construction, strengthens and improves the safety awareness of construction personnel at all levels, and establishes real-time and traceable dynamic engineering archives to bring daily management work to a new level. The information-oriented construction site safety monitoring system provides a powerful technical means for improving the work efficiency of site management personnel and can bring unlimited benefits with limited investment. The present research uses the BP neural network data fusion method based on the multisource data fusion method to build a model to monitor the slope construction of the expressway.

In this paper, the method of multisource data fusion is used to determine the slope parameters based on the slope 
characteristics of an expressway. In situ monitoring of slope displacement and soil parameter inversion is used to determine the soil parameter values, and then the slope is calculated based on the inverse soil parameter values to research on the influence of slope design parameters and treatment measures on slope stability during construction in unexcavated sections.

This study uses a multisource data fusion method to invert the soil parameters to establish a monitoring pointslope model analyze and calculate the displacement values at different depths at the monitoring points and compare with the measured displacement values. The maximum error is $7.53 \%$, less than $10 \%$. The results of soil parameters are reasonable and can be used for the subsequent analysis of the stability of unexcavated slopes. In addition, this paper also analyzes the horizontal surface displacement data and concludes that the slope stability is in line with the actual situation, and the feasibility of this method is verified again. The research results in this paper play an important paradigm role in the construction safety monitoring of future expressways.

\section{Data Availability}

No data were used to support this study.

\section{Conflicts of Interest}

There are no potential conflicts of interest in the paper. All the authors have read the manuscript and approved to submit it to the journal.

\section{Acknowledgments}

This work was supported by National Key R\&D Program of China (no. 2017YFC0805303).

\section{References}

[1] Y. Wang, L. Wang, and C. Wang, "How eye movement and driving performance vary before, during, and after entering a long expressway tunnel: considering the differences of novice and experienced drivers under daytime and nighttime conditions[J]," SpringerPlus, vol. 5, no. 1, p. 538, 2016.

[2] C.-G. Qi, J.-H. Zheng, and D.-J. Zuo, "Method to reduce bump at bridge approach of open expressway using horizontal drilling replacement," Geotechnical \& Geological Engineering, vol. 35, no. 3, pp. 1-8, 2016.

[3] D. Cheng and X. Fu, "Change characteristics and forecast research of road surface temperature on huyu expressway(hubei section)," Meteorological and Environmental Research, vol. 8, no. 5, pp. 92-99, 2017.

[4] H.-F. Jia, Y.-X. Li, and L.-L. Yang, "Expressway traffic joint control based on traffic state estimation," Journal of Jilin University, vol. 47, no. 1, pp. 76-81, 2017.

[5] Y. Huang, Y.-B. Li, and Y. Hong, "The land use change and landscape pattern response driven by YU-YI expressway(chongqing section)," Journal of Natural Resources, vol. 30, no. 9, pp. 1449-1460, 2015.

[6] D.-W. Wang and P. Shen, "Support mechanism and effect of cutting high slope in expressway reconstruction and expansion projects," Changan Daxue Xuebao, vol. 37, no. 3, pp. 39-46, 2017.

[7] H.-B. Wu, "Program feasibility numerical analysis of rail transport beneath expressway and the study of site construction control," Value Engineering, vol. 39, no. 2, pp. 344-352, 2015.

[8] J. Hsi, C. Gunasekara, and V. Nguyen, "Chapter 24 Characteristics of soft peats, organic soils and clays," Elsevier GeoEngineering Book Series, Colombo-Katunayake Expressway, vol. 3, no. 5, pp. 681-722, Sri Lanka, 2015.

[9] G.-L. Yang, Q. Shen, and J.-N. Xu, "Site test and numerical analysis on reinforced earth retaining wall with green gabion on expressway," Zhongguo Gonglu Xuebao/china Journal of Highway \& Transport, vol. 28, no. 6, pp. 18-23, 2015.

[10] M. A. Yue-mei and X.-G. Zhou, "Gradation ratio design and application of micro-surfacing mixtures in tanbai expressway pavement," Western China Communications Science \& Technology, vol. 59, no. 1, pp. 123-142, 2015.

[11] U. Okumura, T. Han, and H. Zhu, "Application of the digital image technology in the visual monitoring and prediction of shuttering construction safety," IOP Conference Series Earth and Environmental Science, vol. 128, no. 1, Article ID 012059, 2018.

[12] Z. G. Zhu, "Research on construction technology of first pile in an urban expressway under complicated conditions," IOP Conference, vol. 100, no. 1, Article ID 012182, 2017.

[13] Y. Wang, X. Jiang, and D. Li, "The robust fusion of multisource gravity data based on the spherical cap harmonic model," Acta Geodaetica Et Cartographica Sinica, vol. 44, no. 9, pp. 952-957, 2015.

[14] L. Rui, Y. Zhang, and P. Zhang, "Location-dependent sensing data collection and processing mechanism in vehicular network," Transactions on Emerging Telecommunications Technologies, vol. 30, no. 11, Article ID e3283, 2018.

[15] M. Zhang and W. Li, "Collaborative classification of hyperspectral and visible images with convolutional neural network," Journal of Applied Remote Sensing, vol. 11, no. 4, p. 1, 2017.

[16] Y. Huang, T. Li, and C. Luo, "Dynamic fusion of multisource interval-valued data by fuzzy granulation," IEEE Transactions on Fuzzy Systems, vol. 26, no. 6, 2018.

[17] G. Xiong, F. Zhu, and X. Dong, "A kind of novel ITS based on space-air-ground big-data," IEEE Intelligent Transportation Systems Magazine, vol. 8, no. 1, pp. 10-22, 2016.

[18] T. Guo, B. Guo, and Yi Ouyang, "CrowdTravel: scenic spot profiling by using heterogeneous crowdsourced data," Journal of Zhejiang University, vol. 9, no. 5, pp. 1-10, 2017.

[19] S. Dong, Z. Yuan, and C. Gu, "Research on intelligent agricultural machinery control platform based on multi-discipline technology integration," Transactions of the Chinese Society of Agricultural Engineering, vol. 33, no. 8, pp. 1-11, 2017.

[20] K. Liu, W. Sheng, and D. Zhang, "Big data application requirements and scenario analysis in smart distribution network," Proceedings of the CSEE, vol. 35, no. 2, pp. 287-293, 2015.

[21] Y. Xu, Bo Du, and L. Zhang, "Advanced multi-sensor optical remote sensing for urban land use and land cover classification: outcome of the 2018 IEEE GRSS data fusion contest," IEEE Journal of Selected Topics in Applied Earth Observations and Remote Sensing, vol. 12, no. 6, pp. 1-16, 2019.

[22] C. Zhang, X. Yang, and H. A. O. Lina, "Lithological classification by synergizing hyperspectral Hyperion and high resolution WorldView-2 satellite images," Journal of Chengdu University of Technology, vol. 44, no. 5, pp. 613-622, 2017. 
[23] J. Shao, "Design of textile manufacturing execution system based on big data," Journal of Mechanical Engineering, vol. 51, no. 5, p. 160, 2015.

[24] K. Alexiev, G. Shishkov, and N. Popova, "Human activity registration using multisensor data fusion," Cybernetics and Information Technologies, vol. 15, no. 7, pp. 99-108, 2015.

[25] Y.-C. Shi, G.-J. Yang, and X.-C. Li, "Intercomparison of the different fusion methods for generating high spatial-temporal resolution data," Journal of Infrared \& Millimeter Waves, vol. 34, no. 1, pp. 92-99, 2015.

[26] W. Liao, F. Van Coillie, and L. Gao, "Deep learning for fusion of APEX hyperspectral and full-waveform LiDAR remote sensing data for tree species mapping," IEEE Access, vol. 6, no. 6, pp. 68716-68729, 2018.

[27] X.-F. Gong, Q.-H. Lin, F.-Y. Cong, and L. De Lathauwer, "Double coupled canonical polyadic decomposition for joint blind source separation," IEEE Transactions on Signal Processing, vol. 66, no. 13, pp. 3475-3490, 2018. 